\title{
Deciphering the mode of action of pollutants impairing the fish larvae escape response with the Vibrational Startle Response
}

\section{Assay}

Melissa Faria ${ }^{1}$, Juliette Bedrossiantz ${ }^{1}$, Eva Prats ${ }^{2}$, Xavier Rovira Garcia ${ }^{1}$, Cristian Gómez-Canela ${ }^{3}$, Benjamin Piña ${ }^{1}$, Demetrio Raldúa'.

${ }^{1}$ IDAEA-CSIC, Jordi Girona 18, 08034, Barcelona, Spain;

${ }^{2}$ CID-CSIC, Jordi Girona 18, 08034, Barcelona, Spain;

${ }^{3}$ Department of Analytical Chemistry and Applied (Chromatography section),

School of Engineering, Institut Químic de Sarrià-Universitat Ramon Llull, Via Augusta 390, 08017 Barcelona, Spain.

${ }^{*}$ Correspondence author e-mail address: mdfaam@cid.csic.es 


\section{Abstract}

The escape response evoked by vibrational stimuli and its habituation, essential behaviors for fish larvae survival, can be altered by neurotoxic environmental pollutants commonly found in our aquatic ecosystems.

In this study we have analyzed the suitability of the Vibrational Startle Response Assay (VSRA) to obtain mechanistic information about the mode of action (MoA) of the chemicals impairing the escape response and its habituation. As a proof of concept, the pathophysiological mechanisms behind the action of two common neurotoxic pesticides, chlorpyrifos-oxon (CPO) and imidacloprid, over their effects on arousal and habituation of the escape response were studied by using pharmacological antagonists of the nicotinic and muscarinic acetylcholine receptors, mecamylamine (MCA) and scopolamine, respectively. Furthermore, potential changes in the neurotransmitter profile were analyzed. Results revealed that whereas the effect of CPO on arousal was mainly mediated by the activation of nAChRs, its effect on habituation was mainly mediated by mAChRs. On the other hand, imidacloprid only affected larvae arousal which was found to be mediated by a cholinergic independent mechanism. No association between behavioral effects on arousal or habituation in affected larvae was found with their corresponding neurotransmitter profile. These results confirm the suitability of VSRA to provide mechanistic information about the potential MoA of neuroactive compounds.

Keywords:

zebrafish, pesticide, startle and habituation, cholinergic modulation 


\section{Highlights:}

- Mechanistic mode of action of neuroactive chemicals was investigated

- The Vibrational Startle Response Assay was applied using zebrafish larvae

- Two neurotoxic pesticides, chlorpyrifos-oxon and imidacloprid, were studied

- Chlorpyrifos-oxon's toxicity was mediated by the activation of both $m$ and nAChRs

- Effect of imidacloprid was not mediated by a cholinergic pathway 


\section{Introduction}

Predation is one of the main sources of mortality of either marine or freshwater fish at all life stages, but particularly for fish larvae (Fuiman and Magurran 1994; Houde and Hoyt 1987). Escape response is a behavioral reflex promoting larval survival to predator's strikes (Anderson et al. 2019; McHenry et al. 2009; Rearick et al. 2018; Zhou and Weis 1998). However, unnecessary escape responses have a high energetic cost and increase the risk of predation, so habituation of this response to irrelevant stimuli results is also essential for survival (Batabyal et al. 2017; Fields and Yen 1997; Killen and Brown 2006).

It has been estimated that up to $30 \%$ of all commercially used chemicals $(\sim 30,000$ chemicals) may have neurotoxic potential (Legradi et al. 2018). Neuroactive chemicals, including neurotoxic pesticides, pharmaceuticals and illicit drugs, are the largest group of micropollutants present in European rivers, where nearly $30 \%$ of all detected chemicals were linked to neurotoxicity (Busch et al. 2016). Furthermore it has been shown that fish larvae behavior, including startle response and its habituation may be disrupted by environmental chemicals (Best et al. 2008; Faria et al. 2019; Kinch et al. 2015; Sandahl et al. 2005; Thompson et al. 2017).

Recently we developed the Vibrational Startle Response Assay (VSRA), an automated in vivo assay for identifying chemicals impairing the escape response and its habituation in zebrafish larvae (Faria et al. 2019). The assay is based on measuring the distance moved by the larva during the startle responses evoked by repetitive vibrational stimuli. The magnitude of the response to the first vibrational stimulus allows evaluating the level of arousal or alertness of the larvae, whereas 
the decrease in the motor response resulting from repeated exposure to the same vibrational stimuli provides information on the habituation process of this response in the larvae. By using VSRA the effect of different concentrations of chlorpyrifosoxon (CPO) and imidacloprid, two neurotoxic pesticides targeting the cholinergic system, on the escape response was characterized.

As a follow-up of our previous work, in this study we intend to test the VSRA's suitability for obtaining mechanistic information about the MoA of the chemicals impairing the escape response of zebrafish larvae. As a proof of principle, the potential involvement of nicotinic and muscarinic acetylcholine receptors ( $\mathrm{n}$ - and mAChRs) in the impairment of the escape response of CPO and imidacloprid were analyzed. A first set of experiments combining pharmacological modulators of specific mammalian $\mathrm{n}$ - and mAChRs (agonists and antagonists) was performed to test the suitability of detecting behavioral modulation with the VSRA. The second set of experiments, aimed to address the potential rescue of the behavioral phenotype induced by each pesticide, was evaluated by co-exposure with mecamylamine (MCA) and scopolamine, pharmacological antagonists of $n$ - and mAChRs, respectively. Finally, potential changes in the neurotransmitter profile associated with the observed neurobehavioral features were examined. The results of this study support the suitability of VSRA not only for screening environmental pollutants impairing arousal and/or habituation, but also to decipher the potential mechanisms of action behind these effects.

\section{Methods}




\subsection{Fish husbandry and larvae production}

Adult wild-type zebrafish, purchased from Piscicultura Superior SL, Parets del Vallès, Barcelona, were maintained in fish water [reverse-osmosis purified water containing $90 \mu \mathrm{g} / \mathrm{mL}$ of Instant Ocean (Aquarium Systems, Sarrebourg, France) and $0.58 \mathrm{mM} \mathrm{CaSO}_{4} \cdot 2 \mathrm{H}_{2} \mathrm{O}$ ] at $28 \pm 1{ }^{\circ} \mathrm{C}$ in the Research and Development Centre of the Spanish Research Council (CID-CSIC) facilities under standard conditions. Embryos were obtained by in-tank group breeding with a 5:3, female:male ratio per tank. Breeding tanks are homemade and include a solid external tank and an internal plastic net. Embryos deposited in the bottom of the tank were collected using a $3 \mathrm{~mL}$ plastic Pasteur pipette and maintained at a 1 individual $/ \mathrm{mL}$ density in fish water at $28.5^{\circ} \mathrm{C}$ on a 12 light: 12 dark photoperiod. Larvae were not fed during the experimental period. All procedures were approved by the Institutional Animal Care and Use Committees at the CID-CSIC and conducted in accordance with the institutional guidelines under a license from the local government (agreement number 9027).

\subsection{Experimental procedure}

The following compounds were purchased from Sigma-Aldrich (St. Louis, MO, USA): nicotine (CAS 54-11-5); pilocarpine (CAS 54-71-7); scopolamine (CAS 5516-3); and imidacloprid (CAS 138261-41-3). Mecamylamine hydrochloride (MCA; CAS 826-39-1) was purchased from Tocris Bioscience (Minneapolis, MN, USA) and CPO (CAS 5598-15-2) was purchased from Chem Service (West Chester, PA, USA). Whereas stock solutions for nicotine, pilocarpine, imidacloprid, CPO and 
MCA were prepared in dimethylsulfoxide (DMSO) and diluted to the test concentrations in fish water on the day of the experiment, experimental solutions of scopolamine were directly prepared in fish water. Since some chemicals like scopolamine exhibit very low permeability in zebrafish larvae, 1\% DMSO was used to ensure the absorption of the chemicals during the exposure period. Controls were also exposed for $24 \mathrm{~h}$ to fish water with $1 \%$ DMSO.

The following concentrations of each compound were used, according to those determined in Faria et al. (2019), where only the highest concentrations which did not induce toxicity, evaluated as either death, gross morphology and/or swimming impairment or clear decrease in the escape response evoked by the tapping on the plate, in 8 dpf zebrafish larvae after 24 hours of exposure: $25 \mu \mathrm{M}$ nicotine; $80 \mu \mathrm{M}$ pilocarpine; $20 \mu \mathrm{M}$ MCA; $25 \mu \mathrm{M}$ scopolamine; 5, 25 and 50nM CPO and 25 and $50 \mu \mathrm{M}$ imidacloprid.

Before performing the co-exposure experiments with the pesticides, a series of experiments were conducted combining pharmacological agonists and antagonists of AChRs. Thus, in order to test the suitability of this assay to decipher the pharmacological MoA of the tested agonists, 7 days post-fertilization (dpf) zebrafish larvae were treated for $24 \mathrm{~h}$ with the following combinations of the selected compounds: (1) nicotine + MCA; and (2) pilocarpine + scopolamine. VSRA was performed immediately after the exposure period.

Then, co-exposure experiments were conducted with the two selected pesticides and the pharmacological antagonists used above. The selected combinations 
were: (1) CPO + MCA; (2) CPO + scopolamine; (3) imidacloprid + MCA; and (4) imidacloprid + scopolamine.

All the exposures were performed at $28.5^{\circ} \mathrm{C}$ (POL-EKO APARATURA Climatic chamber KK350, Poland) with 12L:12D photoperiod in 48 well plates with 1 larva per well and $1 \mathrm{~mL}$ of medium. After 24 hours exposure, larvae were directly tested in the VSRA without further manipulation. At least two independent experiments were performed where groups of 48 larvae underwent behavioral testing.

Data of single exposures of the above mentioned chemicals, already reported in the previous work were used to compare with co-exposure results.

\subsection{Vibrational startle response assay (VSRA)}

Vibrational startle response assay was performed as described in Faria et al. (2019). Videotracking and the escape response were analyzed using the EthoVision XT 9 software (Noldus, Wageningen, The Netherlands). Trials were performed at $28^{\circ} \mathrm{C}$ with near infrared light. Tapping stimulus was selected at the highest intensity (intensity level: 8 ) and 50 sequences of the vibrational stimuli were delivered at an interstimulus interval (ISI) rate of 1 second. Before delivering the first stimulus, larvae were left in the observation chamber for $15 \mathrm{~min}$ to acclimate. Videos were recorded at 30 frames per second and the VSR was analyzed for each individual larva by measuring the distance moved $(\mathrm{cm})$ over the 1s period after stimulus.

\subsection{Extraction and analysis of neurotransmitters by LC-MS/MS}


Pools of 5 zebrafish larvae were used for the extraction and analysis of neurotransmitters following the protocol described by Gómez-Canela et al. (2018). In brief, pools of larvae were spiked with $500 \mathrm{ng}$ of Internal Standard Mixture (ISM) (see supplementary material section 1.1.1), followed by the addition of $500 \mu \mathrm{L}$ of MeOH: $\mathrm{H}_{2} \mathrm{O}$ (90:10). Three stainless steel beads ( $3 \mathrm{~mm}$ diameter) were placed in each sample and homogenized using a bead mill homogenizer (TissueLyser LT, Qiagen) at 50 oscillations per min during $90 \mathrm{~s}$. Samples were then centrifuged during $20 \mathrm{~min}$ at $13,000 \mathrm{rpm}$ at $4^{\circ} \mathrm{C}$. The supernatant was filtered using $0.20 \mu \mathrm{M}$ PTFE filters (DISMIC -13 JP, Advantec ${ }^{\circledR}$ ) and kept in amber chromatographic vials at $-80^{\circ} \mathrm{C}$ before LC-HRMS analysis. Fifteen neurotransmitters were measured: Acetylcholine (Ach); Serotonin; 5-Hydroxyindoleacetic acid (5-HIAA); 5-Hydroxy-Ltryptophan (5-HTP); Tryptophan (Tryp); Dopamine; L-Phenylalanine; L-Tyrosine; 3,4-Dihydroxyphenylalanine (L-DOPA); 3-Methoxytyramine (3-MT); Epinephrine; Norepinephrine (NE); L-Glutamic acid (Glutamate); -Amminobutyric acid (GABA) and Glycine, using liquid chromatography connected to a triple quadrupole detector (Xevo TQD, Waters, USA) (LC-MS/MS). Target compounds were separated using a Synergi Polar-RP $80 \AA$ column $(250 \mathrm{~mm} \times 4.6 \mathrm{~mm}$ ID, particle size $4 \mu \mathrm{M}$, Phenomenex, Torrance, USA). Additional details can be found in Supplementary Methods Section 1.

\subsection{Data analysis}

Data were analyzed with IBM SPSS 19.0 (Statistical Package 2010, Chicago, IL). Data are presented as the mean \pm SEM of all subjects from 2 independent 
experiments, unless stated otherwise. In this study the original data representation has been modified in order to improve the quality of the information provided on the habituation process. Therefore, behavioral responses are represented as: "Arousal": measured as the total distance moved $(\mathrm{cm})$ in response to the first stimulus and as "Habituation": represented as: 1) plots of distance moved relative to the response to the first stimulus (S1); and 2) area under the curve (AUC) of plots of distance moved relative to the response to the first stimulus.

One-way Analysis of Variance (ANOVA) followed by Tukey's multiple comparison tests were used to compare the level differences of each response among experimental groups. Effects over the variance in behavioral responses of the two factors: contaminant concentrations and the presence of AChR antagonist as well as their interaction were analyzed by two-way ANOVA. The statistical test used for each set of results can be found in the text or in the figure caption. Behavioral responses of controls from each experiment and the previous work (Faria et al. 2019) were not found statistically different $(P>0.05$, Tukey's multiple comparison tests, data not shown).

Levels of neurochemicals in pools were normalized per larva and represented as pg/larvae (Supplementary Material Section 2). Data are presented as the mean \pm SEM of 2 independent experiments. One-way ANOVA followed by Dunnett's multiple comparison tests were used to compare the differences in the level neurochemical of each treatment with regard to the control. Significance was set at $P<0.05$. Control responses in plots and bars graphs represent the mean of all controls. 


\section{Results}

\subsection{Pharmacological AChR modulators}

In order to test the suitability of using pharmacological modulators of mammalian nand mAChRs in zebrafish, a set of experiments were initially performed by combining prototypic agonists and antagonists. Thus, nicotine and MCA were selected as agonist and antagonist of the nicotinic AChRs (nAChRs) respectively, whereas pilocarpine and scopolamine were used as agonist and antagonist of the muscarinic AChRs (mAChRs) respectively.

One-way Analysis of Variance indicated significant effects of both groups of pharmacological modulators of the cholinergic system on the larvae escape response, although the effects were stronger for nAChRs modulators. Whereas nAChRs modulators altered both the arousal and the habituation, only the arousal was affected by the mAChR (Table S4, Supplementary Material). While single exposure to nicotine and MCA had no effects on arousal, the co-exposure of these two nAChR modulators resulted in a mild but significant decrease in arousal (Figure 1A). Pilocarpine significantly increased arousal through the activation of $\mathrm{mAChR}$, as indicated by the full recovery observed during the co-exposure with scopolamine (Figure 1B). Nicotine significantly delayed habituation, represented by the significant increase of AUC values, and this effect was mediated through the activation of nAChRs, as indicated by the recovery observed during the coexposure with the specific antagonist MCA (Figure 1C). However, muscarinic modulators showed no effect over the escape response habituation (Figure 1D).

\subsection{Chlorpyriphos-oxon}


Arousal and habituation of the escape response were dramatically affected by CPO. Despite this, cholinergic antagonists were able to significantly recover most of the affected groups. Two-way ANOVA of arousal from single and combined exposure of different CPO concentrations with the selected $n$ - and mAChR antagonists revealed a significant interaction between CPO concentration and cholinergic antagonists (MCA or scopolamine) (Table 1). On the other hand, no interaction was observed for habituation (Table 1). Independent observation of the factors indicates that CPO concentration affected both arousal and habituation, and that the presence of both cholinergic antagonists was significant for arousal, while for habituation only scopolamine displayed important changes over larvae behavior (Table 1).

CPO increased arousal in a concentration-dependent manner (Fig. 2 A, C and E). Whereas $5 \mathrm{nM}$ CPO had no effect on arousal, 25 and $50 \mathrm{nM}$ increased the magnitude of the first startle at 36 and $180 \%$, respectively. Co-exposure of $25 \mathrm{nM}$ CPO with MCA and scopolamine were able to rescue the effect on arousal. Despite this, scopolamine only induced a partial rescue of the effect of $25 \mathrm{nM}$ and even less of 50nM CPO, while MCA was able to induce the full rescue of both concentrations.

Effect of CPO on habituation was significantly delayed in larvae exposed to 5 and $25 \mathrm{nM}$ CPO (Fig. 2 B and D) and not to 50nM CPO (Fig. 2 F). Similar to arousal, the effect of CPO on habituation seems to involve the activation of both $\mathrm{n}$ - and mAChRs, since behavior recovery could be observed when co-exposing with both 
antagonists (Figure 2B and D). However, scopolamine was more efficient than MCA, fully recovering the normal habituation profile of larvae at 5 and $25 \mathrm{nM}$ CPO (Figure 2D). Curiously, although habituation was unaffected by single exposure to 50nM CPO, it was significantly increased by co-exposure with scopolamine.

\subsection{Imidacloprid}

The effects on arousal and habituation of the escape response of zebrafish larvae exposed to 25 and $50 \mu \mathrm{M}$ imidacloprid are depicted in Figure 3 and Table 1. Both concentrations of imidacloprid induced a significant decrease in arousal, but no effects on habituation were found. In fact, the distance moved by the treated larvae in response to the first tap was $24-28 \%$ lower than that of the control larvae (Fig. 3 $A$ and B). Subsequently, the potential involvement of nicotinic and/or muscarinic pathways in the observed effect were tested by co-exposure with MCA and scopolamine. None of the antagonists were able to rescue the imidacloprid effect (Figure 3 and Table 1), which was confirmed by two-way ANOVA analysis (Table 1) that showed no significant interaction between antagonists and imidacloprid for the arousal $(p=0.008)$. Interestingly, $25 \mu \mathrm{M}$ imidacloprid co-exposed with MCA did seem to have an impact on habituation (Fig. 3C), despite this, no significant interaction was observed in the ANOVA analysis (Table 1).

\subsection{Neurotransmitter profile}

It has been demonstrated that different neurotransmitters, including dopamine, serotonin, acetylcholine or glycine, can modulate the vibrational startle response in vertebrates, including fish. In the present study we intended to assess if the 
observed neurobehavioral changes induced by the exposure to CPO and imidacloprid were linked to changes in the neurotransmitter profile induced by $\mathrm{n}$ - or mAChR agonists. Thus, levels of neurotransmitters, as well as their precursors and degradation products, from different neurotransmitter systems potentially involved in the modulation of the escape response have been determined in pools of larvae control and treated with nicotine, pilocarpine, CPO and imidacloprid. Results of the analyses (Supplementary Table S3) show very limited changes in the neurotransmitter profile after the different treatments. Whereas no changes were found in the levels of norepinephrine, serotonin, glycine, GABA and acetylcholine after any treatment, levels of dopamine slightly increased $(p=0.048)$ after treatment with pilocarpine. Levels of glutamate, however, significantly decreased after the treatment with the highest CPO concentration, both imidacloprid concentrations, and pilocarpine. The heat map of hierarchical cluster analysis, in which the magnitude of the first startle (S1) and habituation (AUC) have been also included, showed the absence of a specific profile of neurotransmitters in larvae with similar behavioral effects on arousal or habituation (Fig. 4). However, as neurochemical analyses have been performed on pools of whole larvae, results should be taken with caution, as many of the analyzed chemicals are expressed also in non-neural tissue.

\section{Discussion}

The aim of the present study was to assess if VSRA is a suitable assay for obtaining mechanistic information about the potential MoA of neuroactive 
compounds when the assay is performed after a pharmacological approach. Initially, pharmacological agonists and antagonists of $\mathrm{n}$ - and mAChRs have been used to assess whether the effects on arousal and/or habituation induced by the agonists were reverted by the corresponding antagonists. We have shown that the decrease in habituation found in larvae treated with nicotine can be only partially rescued by co-exposure with the nAChR antagonist MCA (Fig. 1C). One hypothesis that might explain this occurrence could be due to the differences in absorption, distribution, metabolism and excretion (ADME) of these two chemicals, with $\mathrm{MCA}$ requiring a longer time to reach the target tissues. In fact, it has been reported that pre-exposure to MCA prior to co-exposure is more effective in rescuing the behavioral phenotype than direct co-exposure (Levin et al. 2006). Another hypothesis could be the lack of affinity of MCA to bind to nAChRs activated by nicotine. For example, Papke et al., found in zebrafish, that nicotine had high efficacy but low potency for a7 receptors while MCA presented low potency for blocking $\alpha 7$ receptors (Papke et al. 2012). On the other hand, the significant increase in arousal found in larvae exposed to the muscarinic agonist pilocarpine was fully rescued by co-exposure with the muscarinic antagonist scopolamine (Fig 1B). Indeed, Eddins et al., reported pilocarpine to significantly increase zebrafish larvae startle response (Eddins et al. 2010). Furthermore, modulation of zebrafish behavior by scopolamine has been reported in various studies (Bortolotto et al. 2015; Hamilton et al. 2017; Kim et al. 2010), e.g., zebrafish has been proposed as model species to study anxiolytic effects of scopolamine (Hamilton et al. 2017). Thus, our results confirmed that the VSRA is also a suitable assay to test if the behavioral effects induced by cholinergic 
compounds on arousal and/or habituation are mediated by the specific activation of n- or mAChRs.

$\mathrm{CPO}$, the active metabolite of chlorpyrifos, irreversibly inhibits $\mathrm{AChE}$, resulting in an increase in the levels of acetylcholine in the synaptic clefts and the concomitant overstimulation of AChRs (Faria et al. 2015). The effects of exposure to the same three CPO concentrations used in the present study on arousal and habituation have been recently reported (Faria et al. 2019). In this previous study we found an increase in arousal only for the two higher CPO concentrations. However, a concentration-dependent decrease in habituation, measured by using the AUC values, was reported for all three concentrations. Although the use of AUC values has been proposed as a measure of habituation (Best et al. 2008; Faria et al. 2019), a detailed analysis of the time-course of the responses to the vibrational stimuli clearly shows that for those chemicals inducing a strong modulation of arousal, as $50 \mathrm{nM} \mathrm{CPO}$, the changes found in the AUC values are biased by the first response and do not represent a real effect on the habituation process. To fix this problem we have modified the data treatment, setting to $100 \%$ the distance moved after the first stimulus in each treatment group and calculating the responses to the subsequent stimuli relative to the first one. With this adjustment, data of all treatments were normalized, allowing a more refined comparison of the rate of movement decrease to repeated stimuli exposure across all treatments (for further details see Supplementary Results and Discussion, section 2.2). By using this approach, we have demonstrated here that habituation was equally affected by the two lowest CPO concentrations and unaffected by the highest one (Fig $2 \mathrm{~B}, \mathrm{D}$ and $F$ ). This observed $U$ pattern of response, where effects can be seen at lower 
doses rather than higher, is similar to that reported in zebrafish and mammalian species exposed to nicotine (Levin and Chen 2004; Levin and Simon 1998; Schreiber et al. 2002). Furthermore, we found that the effects of CPO over larvae habituation was mediated through both AChRs, however the outcome by muscarinic modulation by scopolamine was more efficient. On the other hand, similar to habituation, the effect of CPO on the arousal also seemed to involve the activation of both AChRs, as the co-exposure with MCA and scopolamine were able to rescue the effect. However, scopolamine only induced a partial rescue over arousal increase which diminished at the highest concentration of $\mathrm{CPO}$, while MCA was able to induce the full rescue at all affected concentrations. Therefore, the obtained results suggest that nAChRs may play an essential role in the observed effect of CPO on arousal, while mAChRs could be more relevant for the effect on habituation. Consistently, Best et al. (2008) reported that the increase in arousal and decrease in habituation found in zebrafish larvae exposed to donepezil, a reversible AChE inhibitor used in human medicine, was mediated by nAChRs. However, in contrast to our results on CPO, these authors found that MCA, but not atropine, was able to fully rescue the effects of donezepil. The fact that scopolamine is more potent than atropine on CNS (Renner et al. 2005) could explain, at least partially, the observed differences of these drugs on the effect of AChE inhibitors.

Imidacloprid, the most widely used neonicotinoid insecticide in the world, binds and activates nAChRs, exhibiting a much greater affinity for insect than for vertebrate receptors (Tomizawa and Casida 2005). However, different reports have shown the toxicity of this insecticide towards non-target species including vertebrates, 
such as birds and rats (Abu Zeid et al. 2019; Kapoor et al. 2014). In the present study, both $\mathrm{n}$ - and mAChRs failed in rescuing the imidacloprid effect on arousal (Fig 3), suggesting the possibility of a non-cholinergic mechanism behind this effect. Interestingly, it has been recently demonstrated that imidacloprid is also a GABA receptor antagonist in insect nervous system (Déglise et al. 2002; Taylor-Wells et al. 2015).

Mauthner cells ( $\mathrm{M}$ cells) play a central role in the vibrational escape response and its habituation in fish (Burgess and Granato 2007). The chemical map of the dendrites of these cells has been characterized and includes AMPA, NMDA, GABA, glycine, dopamine and serotonin receptors (Korn and Faber 2005). Thus, the effects of cholinergic compounds on the escape response and its habituation could involve changes in the neurotransmitter profile of the larvae. However, our results show that changes in arousal and/or habituation after exposure to cholinergic agonists CPO and imidacloprid are not related with changes in the neurotransmitter profile of the whole larvae. By analyzing the neurotransmitter profile in the whole larvae we are integrating potential effects on the central and peripheral nervous system, but at the same time we undergo a loss of sensitivity to small changes in specific areas of the CNS. However, our results may reflect the complexity of the mechanisms involved in the modulatory action of these chemicals.

In summary, the results presented in this manuscript demonstrate that the recently developed VSRA can be a useful tool for obtaining mechanistic information about the potential MoA of neuroactive compounds when the assay is performed after a pharmacological approach. 


\section{Acknowledgments}

This work was supported by the Spanish Government (CTM2017-83242-R) and by the NATO SfP project MD.SFPP 984777. M.F acknowledges financial support from the Beatriu de Pinós programme (grant №: 2016 BP 00233) provided by the Secretariat of Universities and Research department of the Ministry for Business and Knowledge, Catalonia Government. J.B acknowledges financial support from Bouygues Fundation's bursary, in the frame of the Erasmus Traineeship (FTOULOUS03). 


\section{References}

Abu Zeid EH, Alam RTM, Ali SA, Hendawi MY (2019) Dose-related impacts of imidacloprid oral intoxication on brain and liver of rock pigeon (Columba livia domestica), residues analysis in different organs. Ecotoxicology and Environmental Safety

167:60-68 doi:https://doi.org/10.1016/j.ecoenv.2018.09.121

Anderson PJ, Mitchell MD, Fedoroff KJ, Chivers DP, Ferrari MC (2019) The Effects of Selenomethionine on the Escape Behaviours of Fathead Minnows. Archives of environmental contamination and toxicology:1-6

Batabyal A, Balakrishna S, Thaker M (2017) A multivariate approach to understanding shifts in escape strategies of urban lizards. Behavioral Ecology and Sociobiology 71(5):83 doi:10.1007/s00265-017-2307-3

Best JD, Berghmans S, Hunt JJ, et al. (2008) Non-associative learning in larval zebrafish. Neuropsychopharmacology 33(5):1206

Bortolotto JW, de Melo GM, de Paula Cognato G, Vianna MRM, Bonan CD (2015) Modulation of adenosine signaling prevents scopolamine-induced cognitive impairment in zebrafish. Neurobiology of learning and memory 118:113-119

Burgess HA, Granato M (2007) Sensorimotor gating in larval zebrafish. Journal of Neuroscience 27(18):4984-4994

Busch W, Schmidt S, Kühne R, Schulze T, Krauss M, Altenburger R (2016) Micropollutants in European rivers: A mode of action survey to support the development of effect_based tools for water monitoring. Environmental toxicology and chemistry 35(8):1887-1899

Déglise P, Grünewald B, Gauthier M (2002) The insecticide imidacloprid is a partial agonist of the nicotinic receptor of honeybee Kenyon cells. Neuroscience letters 321(1-2):13-16

Eddins D, Cerutti D, Williams P, Linney E, Levin ED (2010) Zebrafish provide a sensitive model of persisting neurobehavioral effects of developmental 
chlorpyrifos exposure: comparison with nicotine and pilocarpine effects and relationship to dopamine deficits. Neurotoxicology and teratology 32(1):99-108

Faria M, Garcia-Reyero N, Padrós F, et al. (2015) Zebrafish models for human acute organophosphorus poisoning. Scientific reports 5:15591

Faria M, Prats E, Novoa-Luna KA, et al. (2019) Development of a vibrational startle response assay for screening environmental pollutants and drugs impairing predator avoidance. Science of The Total Environment 650:87-96

Fields DM, Yen J (1997) The escape behavior of marine copepods in response to a quantifiable fluid mechanical disturbance. Journal of Plankton Research 19(9):1289-1304

Fuiman LA, Magurran AE (1994) Development of predator defences in fishes. Reviews in Fish Biology and Fisheries 4(2):145-183

Gómez-Canela C, Tornero-Cañadas D, Prats E, Piña B, Tauler R, Raldúa D (2018) Comprehensive characterization of neurochemicals in three zebrafish chemical models of human acute organophosphorus poisoning using liquid chromatography-tandem mass spectrometry. Analytical and bioanalytical chemistry 410(6):1735-1748

Hamilton TJ, Morrill A, Lucas K, et al. (2017) Establishing zebrafish as a model to study the anxiolytic effects of scopolamine. Scientific Reports 7(1):15081 doi:10.1038/s41598-017-15374-w

Houde E, Hoyt R (1987) Fish early life dynamics and recruitment variability. Trans Am Fish Soc

Kapoor U, Srivastava MK, Trivedi P, Garg V, Srivastava LP (2014) Disposition and acute toxicity of imidacloprid in female rats after single exposure. Food and Chemical Toxicology 68:190-195 doi:https://doi.org/10.1016/j.fct.2014.03.019

Killen SS, Brown JA (2006) Energetic cost of reduced foraging under predation threat in newly hatched ocean pout. Marine Ecology Progress Series 321:255-266

Kim Y-H, Lee Y, Kim D, Jung MW, Lee C-J (2010) Scopolamine-induced learning impairment reversed by physostigmine in zebrafish. Neuroscience Research 67(2):156-161 doi:https://doi.org/10.1016/j.neures.2010.03.003 
Kinch CD, Ibhazehiebo K, Jeong J-H, Habibi HR, Kurrasch DM (2015) Low-dose exposure to bisphenol $A$ and replacement bisphenol $S$ induces precocious hypothalamic neurogenesis in embryonic zebrafish. Proceedings of the National Academy of Sciences 112(5):1475-1480

Korn H, Faber DS (2005) The Mauthner cell half a century later: a neurobiological model for decision-making? Neuron 47(1):13-28

Legradi JB, Di Paolo C, Kraak MHS, et al. (2018) An ecotoxicological view on neurotoxicity assessment. Environmental Sciences Europe 30(1):46 doi:10.1186/s12302-018-0173-x

Levin ED, Chen E (2004) Nicotinic involvement in memory function in zebrafish. Neurotoxicology and Teratology 26(6):731-735 doi:https://doi.org/10.1016/j.ntt.2004.06.010

Levin ED, Limpuangthip J, Rachakonda T, Peterson M (2006) Timing of nicotine effects on learning in zebrafish. Psychopharmacology 184(3):547-552 doi:10.1007/s00213-005-0162-9

Levin ED, Simon BB (1998) Nicotinic acetylcholine involvement in cogitive function in animals. Psychopharmacology 138(3-4):217-230 doi:10.1007/s002130050667

McHenry M, Feitl K, Strother J, Van Trump W (2009) Larval zebrafish rapidly sense the water flow of a predator's strike. Biology Letters 5(4):477-479

Papke RL, Ono F, Stokes C, Urban JM, Boyd RT (2012) The nicotinic acetylcholine receptors of zebrafish and an evaluation of pharmacological tools used for their study. Biochemical Pharmacology 84(3):352-365 doi:https://doi.org/10.1016/i.bcp.2012.04.022

Rearick DC, Ward J, Venturelli P, Schoenfuss H (2018) Environmental oestrogens cause predation-induced population decline in a freshwater fish. Royal Society open science 5(10):181065

Renner UD, Oertel R, Kirch W (2005) Pharmacokinetics and pharmacodynamics in clinical use of scopolamine. Therapeutic drug monitoring 27(5):655-665

Sandahl JF, Baldwin DH, Jenkins JJ, Scholz NL (2005) Comparative thresholds for acetylcholinesterase inhibition and behavioral impairment in coho salmon 
exposed to chlorpyrifos. Environmental Toxicology and Chemistry: An International Journal 24(1):136-145

Schreiber R, Dalmus M, De Vry J (2002) Effects of a $4 / \beta$ 2-and a 7-nicotine acetylcholine receptor agonists on prepulse inhibition of the acoustic startle response in rats and mice. Psychopharmacology 159(3):248-257

Taylor_Wells J, Brooke BD, Bermudez I, Jones AK (2015) The neonicotinoid imidacloprid, and the pyrethroid deltamethrin, are antagonists of the insect Rdl GABA receptor. Journal of neurochemistry 135(4):705-713

Thompson WA, Arnold VI, Vijayan MM (2017) Venlafaxine in embryos stimulates neurogenesis and disrupts larval behavior in zebrafish. Environmental science \& technology 51(21):12889-12897

Tomizawa M, Casida JE (2005) Neonicotinoid insecticide toxicology: mechanisms of selective action. Annu Rev Pharmacol Toxicol 45:247-268

Zhou T, Weis J (1998) Swimming behavior and predator avoidance in three populations of Fundulus heteroclitus larvae after embryonic and/or larval exposure to methylmercury. Aquatic Toxicology 43(2-3):131-148 


\section{Author contributions}

M.F and E.P performed all the exposure experiments; M.F and J.B performed the behavioral analyses; C.G.C, X.R.G and M.F preformed neurotransmitter analyses; B.P, M.F and D.R. were involved in the interpretation of the data. M.F and D.R. were involved in the conception, design and writing of the manuscript.

\section{Competing financial interests}

The authors declare no competing financial interest. 
Table 1. Two way ANOVA assessing effects of contaminant concentrations, $n$ or mAChR antagonist, and the interaction of both, over the variance of zebrafish larvae behavioural responses by. $\mathrm{P}<0.05$.

\begin{tabular}{|c|c|c|c|c|c|c|c|c|c|}
\hline & \multicolumn{3}{|c|}{ Concentration (Con) } & \multicolumn{3}{|c|}{ Antagonist (Ant) } & \multicolumn{3}{|c|}{ Con x Ant (interaction) } \\
\hline & df & $F$ & $\mathrm{P}$ & $\mathrm{df}$ & $\mathrm{F}$ & $P$ & $\mathrm{df}$ & $\mathrm{F}$ & $P$ \\
\hline \multicolumn{10}{|l|}{ Startle response } \\
\hline CPO+MCA & 3,326 & 44.530 & 0.000 & 1,326 & 76.088 & 0.000 & 2,326 & 34.641 & 0.000 \\
\hline Imidacloprid+MCA & 2,234 & 24.429 & 0.000 & 1,234 & 7.170 & 0.008 & 1,234 & 0.012 & 0.913 \\
\hline Imidacloprid+Scopolamine & 2,231 & 27.521 & 0.000 & 1,231 & 3.703 & 0.056 & 1,231 & 0.415 & 0.520 \\
\hline \multicolumn{10}{|l|}{$\underline{A \cup C}$} \\
\hline $\mathrm{CPO}+\mathrm{MCA}$ & 3,299 & 5.869 & 0.001 & 1,299 & 1.925 & 0.166 & 2,299 & 2.266 & 0.106 \\
\hline CPO+Scopolamine & 3,306 & 11.280 & 0.000 & 1,306 & 17.882 & 0.000 & 2,306 & 0.414 & 0.662 \\
\hline Imidacloprid+MCA & 2,229 & 0.289 & 0.749 & 1,229 & 6.702 & 0.010 & 1,299 & 1.896 & 0.170 \\
\hline Imidacloprid+Scopolamine & 2,225 & 0.112 & 0.894 & 1,225 & 1.544 & 0.215 & 1,225 & 1.145 & 0.286 \\
\hline
\end{tabular}




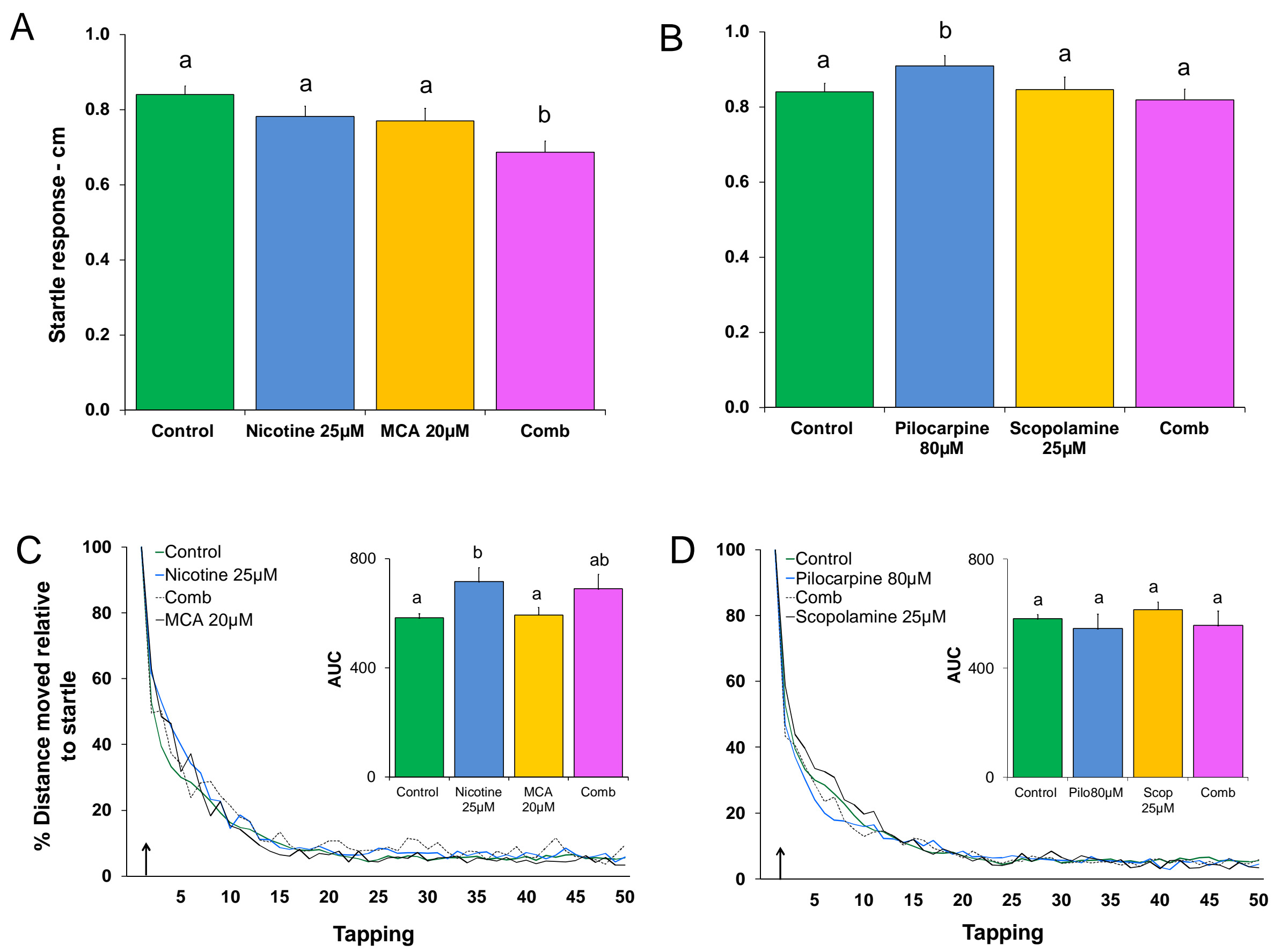
a

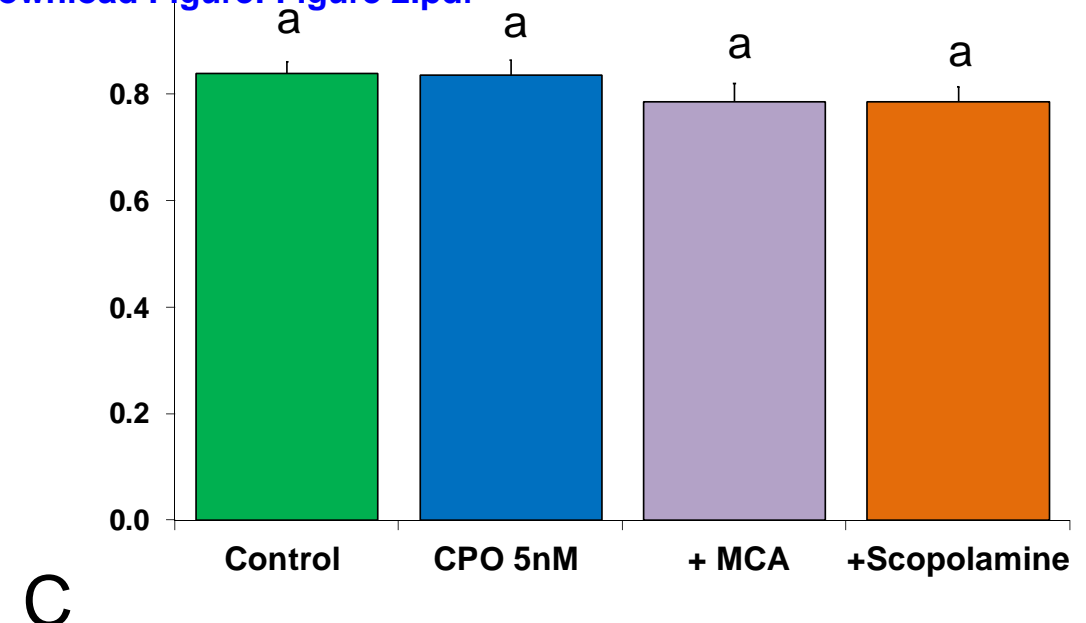

C

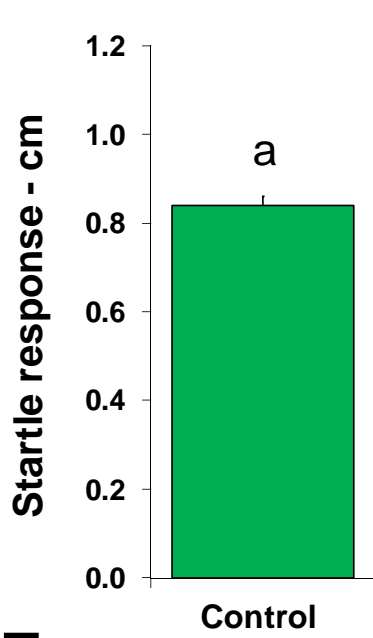

E

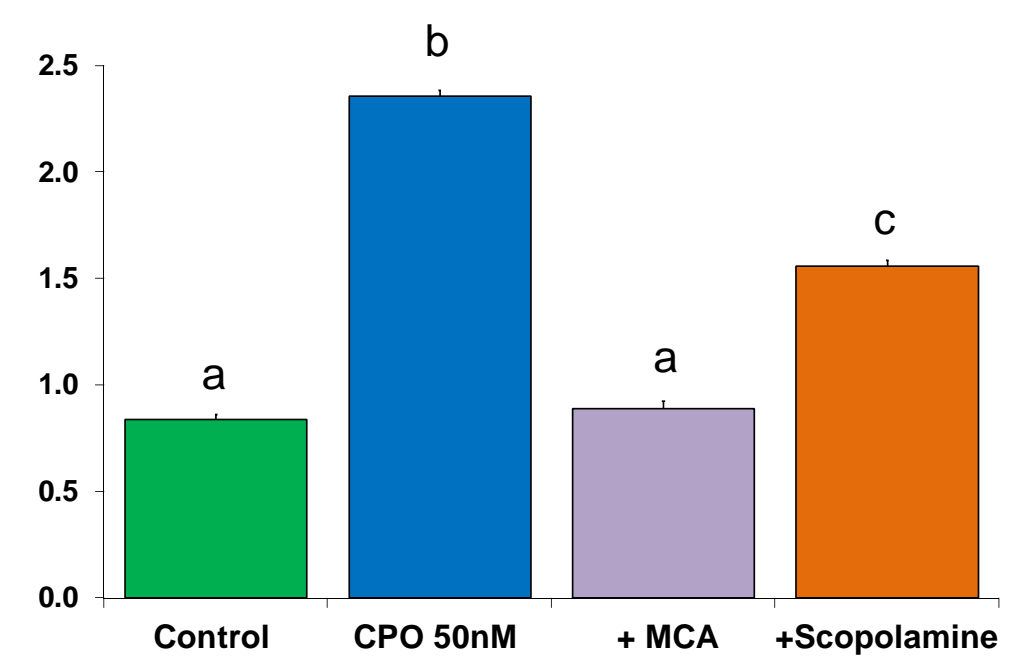

$a b$

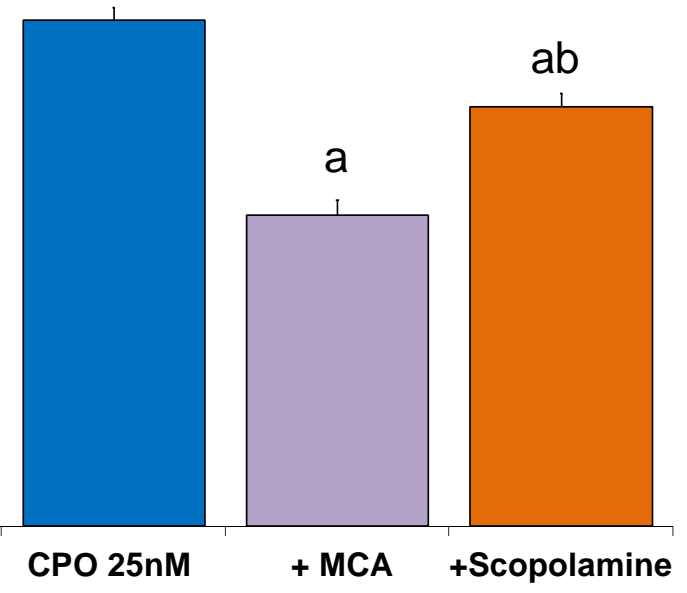

b
F 100$]$-Control
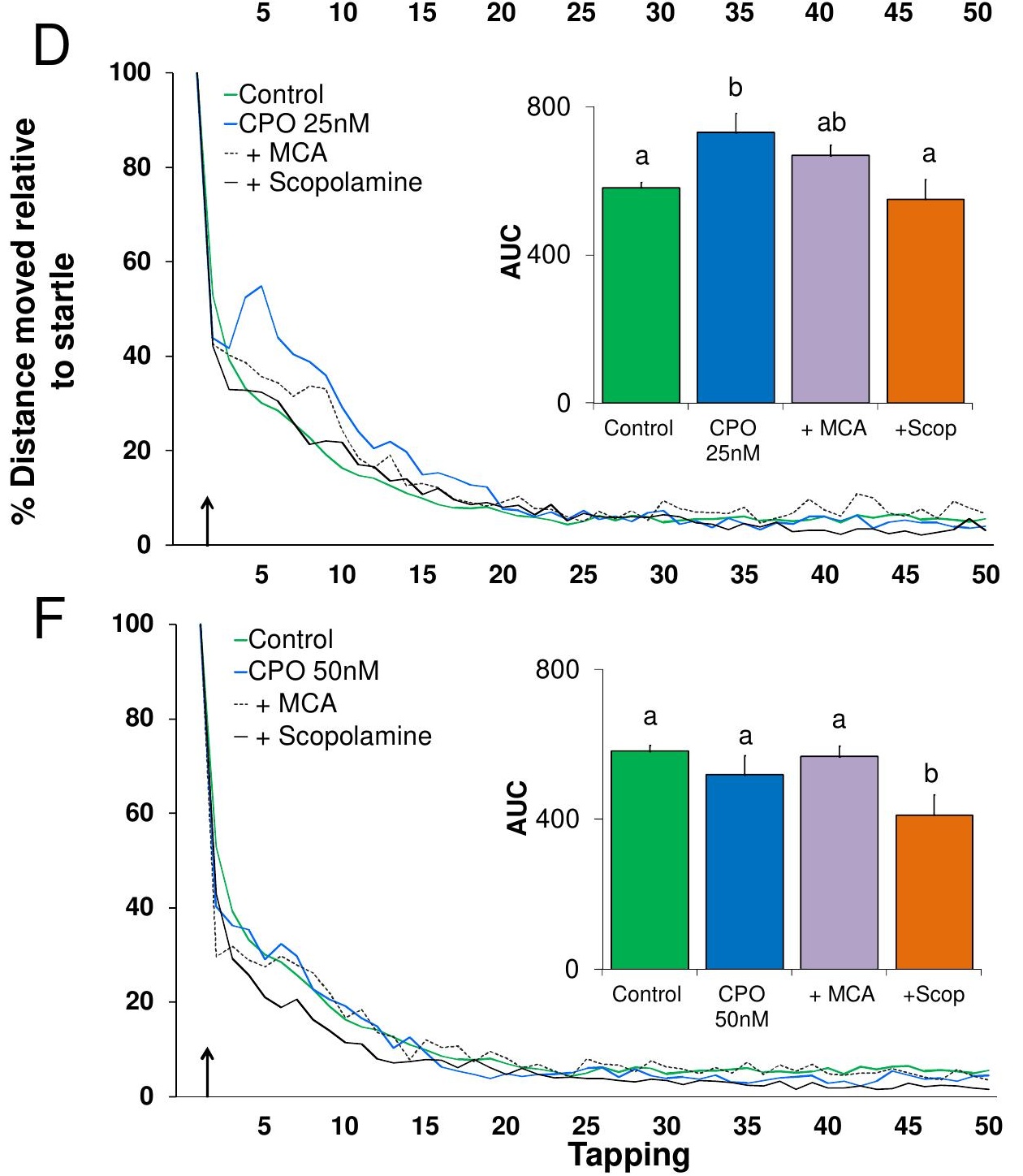

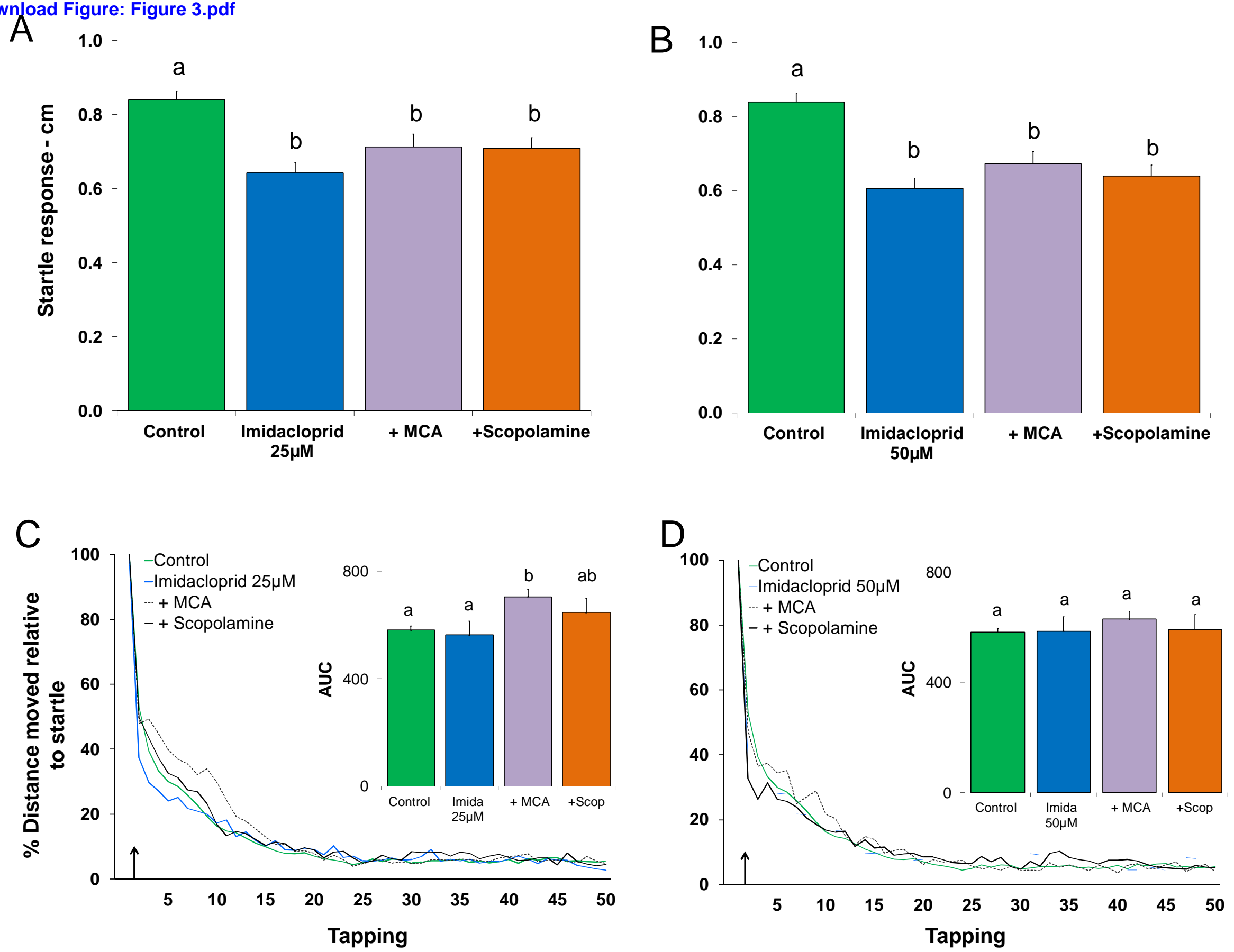
Color Key
Figure: Figure 4.p
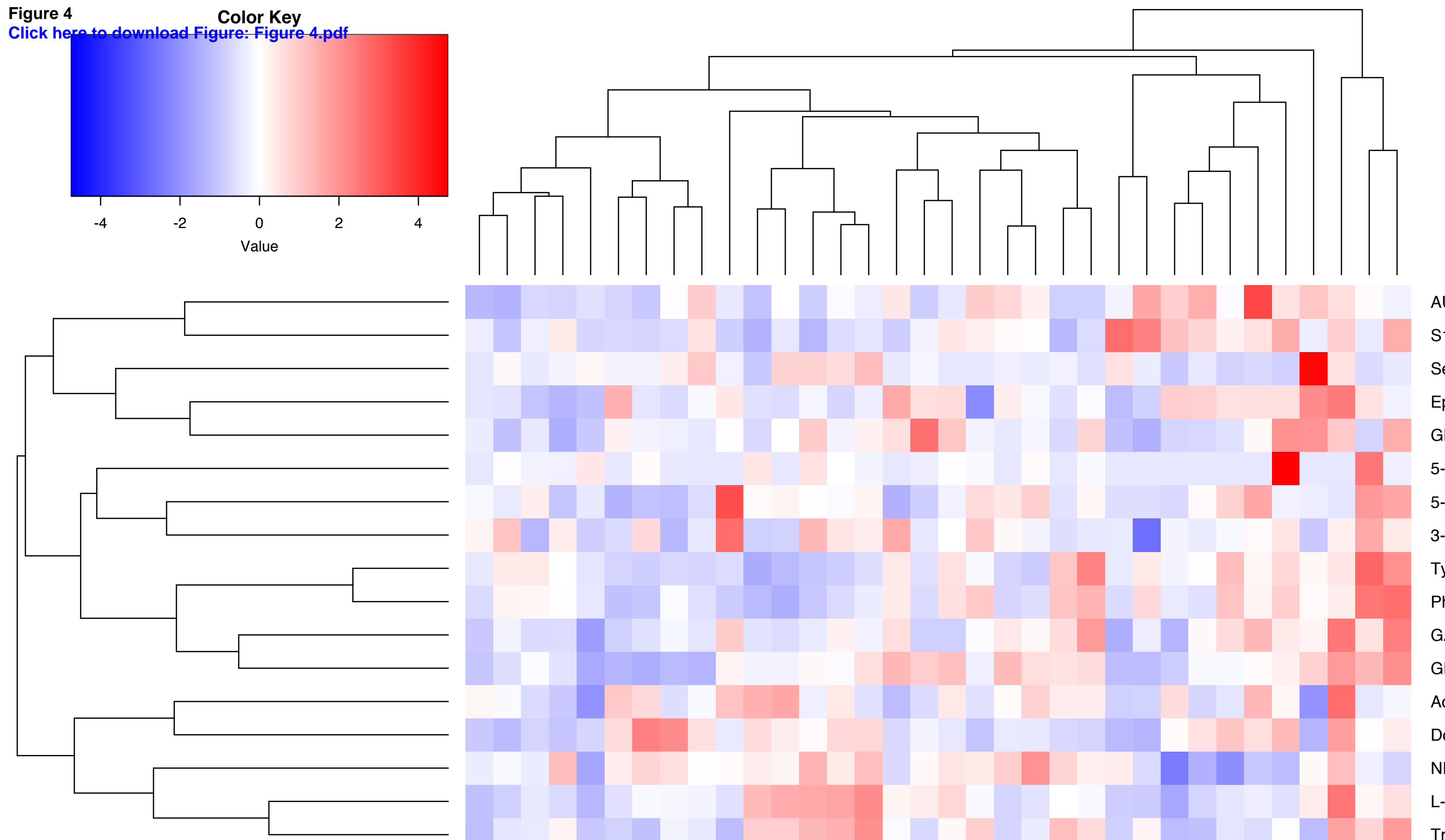

Tyrosine

Phenylalanine

GABA

Glutamate

Ach

Dopamine

NE

L-DOPA

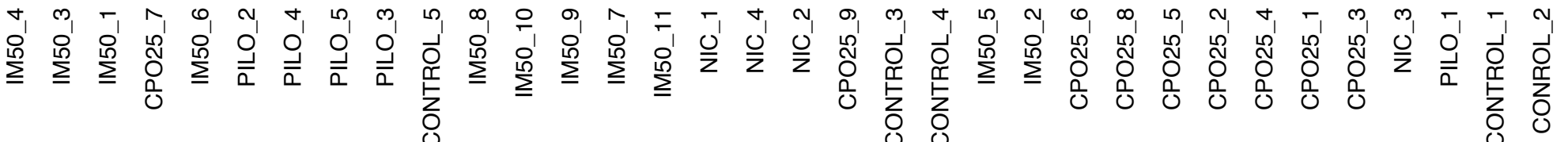




\section{Figure Captions:}

Figure 1 - Zebrafish escape responses and their habituation following pharmacological modulation of nicotinic and muscarinic AChRs. Upper panel: A-B: Distance moved $(\mathrm{cm})$ during the startle response following delivery of the 1st stimulus in larvae from different experimental groups (mean \pm SEM). Lower panel: C-D: Habituation of zebrafish larvae from different experimental groups, measured as the decrease in the distance moved (percentage) across 50 tapping stimuli delivered at an interstimulus interval of $1 \mathrm{~s}$, relative to the response to the first stimulus delivered (set to $100 \%$ ). Black arrow indicates the time of delivery of the first stimulus. AUCs (mean \pm SEM) of each treatment are shown as an inset to each plot. A-C: control $(n=225) / 25 \mu M$ nicotine $(n=89) /$ $20 \mu \mathrm{M}$ MCA $(n=87) / 25 \mu \mathrm{M}$ nicotine $+20 \mu \mathrm{M}$ MCA (Comb) ( $\mathrm{n}=83$ ); B-D: control $(n=225) / 80 \mu M$ pilocarpine $(n=83) / 25 \mu$ M scopolamine $(n=91) / 80 \mu M$ pilocarpine $+25 \mu \mathrm{M}$ scopolamine (Comb) $(\mathrm{n}=91)$. Different letters indicate groups that are statistically similar ( $p>0.05)$ according to Tukey's multiple-comparison test.

Figure 2 - Effects of different concentrations of CPO over zebrafish larvae startle response and its habitation. Left panel (A, C, E): Distance moved (cm) during the startle response following delivery of the 1st stimulus in larvae from different experimental groups (mean \pm SEM). Right panel (B, D, F): Black arrow indicates the time of delivery of the first stimulus. AUCs (mean \pm SEM) of each treatment are shown as an inset to each plot. A-B: Control $(n=225) / 5 n M$ CPO 
$(n=85) / 5 n M C P O+20 \mu M$ MCA $(+M C A)(n=90) /$ and $5 n M C P O+25 \mu M$ scopolamine (+Scop) ( $n=90)$; C-D: Control ( $n=? ?) / 25 n M$ CPO ( $n=79) / 25 n M$ $\mathrm{CPO}+20 \mu \mathrm{M}$ MCA $(+\mathrm{MCA})(\mathrm{n}=85) / 25 \mathrm{nM} \mathrm{CPO}+25 \mu \mathrm{M}$ scopolamine (+Scopolamine) ( $n=87)$; E-F: Control $(n=225) / 50 n M$ CPO $(n=72) / 50$ nM CPO +

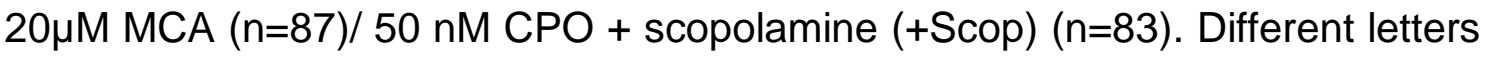
represented in each bar indicate groups that are statistically similar $(p>0.05)$ according to Tukey's multiple-comparison test.

Figure 3 - Effects of two concentrations of imidacloprid over zebrafish larvae startle response and its habitation. Upper panel: A-B: Distance moved $(\mathrm{cm})$ during the startle response following delivery of the 1st stimulus in larvae from different experimental groups (mean \pm SEM). Lower panel: C-D: Black arrow indicates the time of delivery of the first stimulus. AUCs (mean \pm SEM) of each treatment are shown as an inset to each plot. A-C: Control $(n=225) / 25 \mu M$

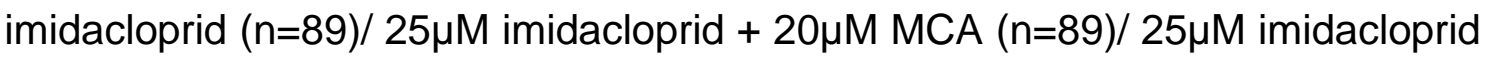
$+25 \mu \mathrm{M}$ scopolamine ( $\mathrm{n}=86)$; B-D: Control $(\mathrm{n}=225) / 50 \mu \mathrm{M}$ imidacloprid $(\mathrm{n}=83) /$ $50 \mu \mathrm{M}$ imidacloprid $+20 \mu \mathrm{M}$ MCA $(\mathrm{n}=89) / 50 \mu \mathrm{M}$ imidacloprid $+25 \mu \mathrm{M}$ scopolamine $(n=88)$. Different letters represented in each bar indicate groups that are statistically similar $(p>0.05)$ according to Tukey's multiple-comparison test.

Figure 4 - Hierarchical clustering of behavioral responses and neurotransmitter concentrations (right of the clustering) in zebrafish larvae after the different treatments (bottom of the clustering). Neurotransmitter responses included: The color scale ranges from blue (low concentration) to red (high concentration, normalized values). Dendrograms corresponding to the hierachical clustering of 
both samples and compounds are shown on the top and on the left of the heatmaps, respectively. Clustering was performed using the gplots package in R. Abbreviations list: AUC - area under the curve; S1 - first startle; IM imidacloprid; NIC - nicotine; PILO - pilocarpine. Neurotransmitter extended name and respective abbreviation can be found in section 2.4. 